\title{
High-Coverage Structures of Carbon Monoxide Adsorbed on Pt(111) Studied by High-Pressure Scanning Tunneling Microscopy ${ }^{\dagger}$
}

\author{
Sarah R. Longwitz,,$\$$ Joachim Schnadt, \\ Erik Lagsgaard, ${ }^{\ddagger}$ Ivan Stensgaard, ${ }^{\ddagger}$ Harald Brune, ${ }^{\S}$ and Flemming Besenbacher*, \\ Interdisciplinary Nanoscience Center (iNANO) and Department of Physics and Astronomy, \\ University of Aarhus, 8000 Aarhus C, Denmark, Institut de Physique des Nanostructures, \\ Ecole Polytechnique Fédérale de Lausanne, 1015 Lausanne, Switzerland
}

Received: February 20, 2004

\begin{abstract}
High-pressure scanning tunneling microscopy was used to study the room-temperature adsorption of CO on a $\operatorname{Pt}(111)$ single-crystal surface in equilibrium with the gas phase. The coverage was found to vary continuously, and over the entire range from $10^{-6}-760$ Torr pressure-dependent moiré patterns were observed, characteristic of a hexagonal or nearly hexagonal $\mathrm{CO}$ overlayer. Two different pressure ranges can be distinguished: below $10^{-2}$ Torr, the moiré lattice vector is oriented along a $30^{\circ}$ high-symmetry direction of the substrate, corresponding to a pressure-dependent rotation of the $\mathrm{CO}$ overlayer with respect to the $(1 \times 1) \mathrm{Pt}$ surface lattice, while above $10^{-2}$ Torr, the $\mathrm{CO}$ layer angle is independent of the pressure. This behavior is analyzed in terms of the interplay of the repulsive $\mathrm{CO}-\mathrm{CO}$ interaction potential and the substrate potential.
\end{abstract}

\section{Introduction}

Few adsorbate systems have received as much attention as carbon monoxide on transition-metal surfaces. The enormous interest originates from the system's relevance to catalysis, since $\mathrm{CO}$ takes part in many important reactions such as $\mathrm{CO}$ hydrogenation and oxidation and the Fischer-Tropsch synthesis ${ }^{1}$ and transition-metal particles are the most common catalytic materials for these reactions. Notwithstanding these beneficial interactions, carbon monoxide is also an unwanted poison molecule in, e.g., the hydrogen feed for low-temperature fuel cells produced from hydrocarbons. ${ }^{2}$ Since all these aspects can be traced back to adsorbate-surface interactions, it is important to acquire a thorough understanding of the properties of the adsorption of CO on transition-metal surfaces.

To date, an impressively detailed knowledge has been gained on the adsorption of $\mathrm{CO}$ on $\mathrm{Pt}(111)$ under ultrahigh vacuum (UHV) conditions (see, e.g., refs 3, 4, and 5). The relevance of this understanding to industrial catalytic conditions has however been questioned. ${ }^{6,7}$ The divergence of experimental and theoretical results for systems under UHV and catalytic or realistic pressures, respectively, has been designated "the pressure gap", which has to be bridged (see, e.g., ref 8). Therefore the development of surface-sensitive techniques that are capable of working at elevated pressures has renewed the interest in the $\mathrm{CO} / \mathrm{Pt}(111)$ system, and a number of recent high-pressure studies have addressed the subject using different techniques. . $, 7,9,10$ These studies revealed that carbon monoxide forms a hexagonal overlayer on $\mathrm{Pt}(111)$ at atmospheric pressure and room temperature. Two different structures were proposed, one close to a $(4 \times 4)-9 \mathrm{CO}$ nonrotated structure, ${ }^{7}$ the other one compatible with a $(\sqrt{19} \times \sqrt{19})$ R23.4० $-13 \mathrm{CO}$ commensurate structure. ${ }^{10}$ The latter structure has also been proposed for the adsorption of $\mathrm{CO}$ in an electrochemical cell at $0-0.2 \mathrm{~V}$ electrode potential. ${ }^{11}$ In contrast, under UHV and low-temperature condi-

\footnotetext{
$\dagger$ Part of the special issue "Gerhard Ertl Festschrift".

* To whom correspondence may be addressed. E-mail: fbe@phys.au.dk.

$\div$ University of Aarhus.

$\S$ Ecole Polytechnique Fédérale de Lausanne.
}

tions, a series of nonhexagonal commensurate structures is observed. ${ }^{3,4,12}$ These are the $c(4 \times 2)$ or $(\sqrt{3} \times 2)$ rect $(\theta=1 / 2$, where $\theta$ is the coverage (ref 13)) and $\mathrm{c}(\sqrt{3} \times 5)$ rect $(\theta=3 / 5)$, $(\sqrt{3} \times 3)$ rect $(\theta=2 / 3)$, and $c(\sqrt{3} \times 7)$ rect $(\theta=5 / 7)$ structures; the latter three can be cast in a unified picture in which unit stripes of the $c(4 \times 2)$ structure separated by domain walls of higher, structure-specific CO density. ${ }^{4}$ It is not yet clear, however, whether these lattice gas structures represent global or local minima of the potential energy surface.

While it was shown that a hexagonal CO overlayer can also be produced under vacuum conditions when the sample is cooled sufficiently, ${ }^{3,10}$ the understanding of the atomic-scale details of the room-temperature adsorption structures of $\mathrm{CO}$ on $\mathrm{Pt}(111)$ over the entire pressure range from UHV pressures up to atmospheric pressure is still incomplete. In particular, no study has been able to unambiguously determine the adsorbate structure for the complete pressure range from UHV to one atmosphere.

For the present study we used high-resolution scanning tunneling microscopy (STM) to unravel the room-temperature adsorption structures of $\mathrm{CO} / \mathrm{Pt}(111)$. At all pressures above $10^{-6}$ Torr, we observed moiré patterns characteristic of the formation of a hexagonal $\mathrm{CO}$ overlayer on the hexagonal $\mathrm{Pt}(111)$ surface. The superposition of the two hexagonal lattices leads to a beating phenomenon resulting in the moiré pattern. A one-dimensional analogy is given by the sum of two sine functions with slightly different frequencies. At low CO pressures, up to $10^{-2}$ Torr, the moiré pattern is characterized by a lattice vector oriented along one of the high-symmetry directions of the substrate, while at higher $\mathrm{CO}$ pressures, a rotation of the pattern is observed. In the following, we discuss these results in the context of existing theories and compare them to available UHV low-temperature data and results obtained in electrochemical cells.

\section{Experimental Section}

The experiments were performed in a UHV chamber equipped with a home-built Aarhus scanning tunneling microscope as well as UHV standard equipment for sample cleaning and charac- 
(a)

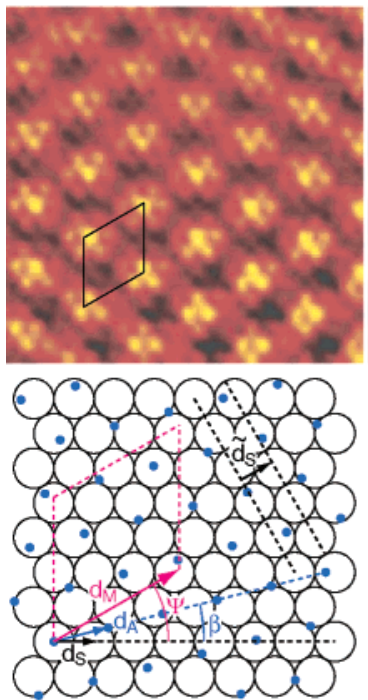

(c) (b)

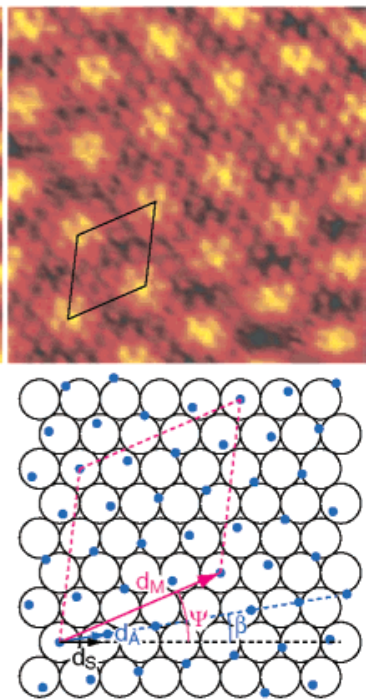

(d)

Figure 1. STM images of $55 \times 55 \AA^{2}$ and corresponding ball models showing moiré superstructures of $\mathrm{CO}$ on $\mathrm{Pt}(111)$ at room temperature. (a) Incommensurate structure at $p=10^{-2}$ Torr, $I_{\mathrm{t}}=1.06 \mathrm{nA}, V_{\mathrm{t}}=8.2$ $\mathrm{mV}$. (b) $p=720$ Torr, $I_{\mathrm{t}}=1.27 \mathrm{nA}, V_{\mathrm{t}}=4.9 \mathrm{mV}$. The images a and $\mathrm{b}$ are aligned so that the bulk [1 $1 \overline{1} 0]$ direction is oriented along the $x$ axis. The image treatment comprised frequency filtering and interpolation. In the ball models in $\mathrm{c}$ and $\mathrm{d}$, the open circles represent Pt atoms and the dark blue dots $\mathrm{CO}$ molecules. The rotational angles $\beta$ between the substrate and adsorbate lattices and $\Psi$ between the substrate and moiré lattices are indicated. The moiré unit cell with lattice constant $d_{\mathrm{M}}$ is also shown. (c) Incommensurate structure at $10^{-2}$ Torr. $\Psi=$ $30^{\circ}, \beta=10.4^{\circ}, d_{\mathrm{M}} / d_{\mathrm{S}}=3.6 . \overrightarrow{\widetilde{d}}_{\mathrm{S}}$ is the real space equivalent of the reciprocal vector $\overrightarrow{\tilde{q}}_{\mathrm{s}}$ observed in the fast Fourier transform (cf. Figure 2). $\overrightarrow{\vec{d}}_{\mathrm{S}}$ is rotated by $30^{\circ}$ from $\vec{d}_{\mathrm{S}}$, and the length relationship is $\tilde{d}_{\mathrm{S}}=$ $\sqrt{3} d_{\mathrm{s}} / 2$. (d) Commensurate $(\sqrt{19} \times \sqrt{19}) \mathrm{R} 23.4^{\circ}-13 \mathrm{CO}$ structure at 720 Torr. $\Psi=23.4^{\circ}, \beta=9.5^{\circ}, d_{\mathrm{M}} / d_{\mathrm{S}}=4.4$.

terization. ${ }^{14}$ Connected to this chamber is a gold-plated cell containing the newly developed Aarhus high-pressure scanning tunneling microscope. ${ }^{15}$ With this, STM experiments can be carried out at pressures of up to 760 Torr of pure gases or gas mixtures. Sample transfer between the systems takes place under clean conditions, i.e., without removing the sample from the vacuum. The gas exposure system of the high-pressure cell has been designed to avoid nickel-carbonyl $\left(\mathrm{Ni}(\mathrm{CO})_{4}\right)$ formation, which is of utmost importance when working with $\mathrm{CO}$. For that purpose, the gas line has been constructed primarily using copper tubing instead of stainless steel. Furthermore, just prior to the inlet, the $\mathrm{CO}$ is passed through an activated high surface area carbon powder filter heated to $\sim 400 \mathrm{~K}$, over which the $\mathrm{Ni}$ carbonyls dissociate, leaving the Ni behind. The efficiency of this system was checked by performing Auger electron spectroscopy after extended high-pressure $\mathrm{CO}$ exposure and subsequent pump-out.

The sample was cleaned in the UHV chamber following standard sample cleaning procedures: $30 \mathrm{~min} 800 \mathrm{eV} \mathrm{Ar}^{+}$ sputtering, followed by an annealing procedure (in case of severely contaminated samples, $10 \mathrm{~min}$ annealing at $800 \mathrm{~K}$ in $10^{-7}$ Torr $\mathrm{O}_{2}$ plus 3 min annealing at $1000 \mathrm{~K}$ in UHV, otherwise just UHV anneal). The experiments at low and medium pressure (up to $5 \times 10^{-4}$ Torr) were then performed in the UHV chamber, while those at higher pressures up to 760 Torr were performed in the high-pressure cell. For pressures between 0.1 and 100 Torr, electric discharges between the closely spaced piezo electrodes may occur. We thus always work at a total pressure of close to 760 Torr in the high-pressure cell. Because of its
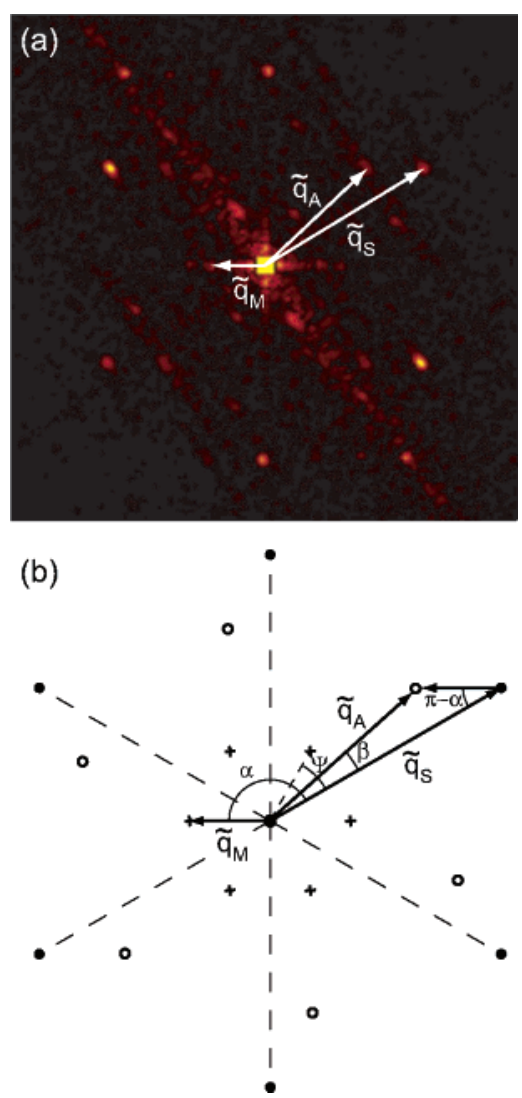

Figure 2. (a) FFT of an STM image of a CO/Pt(111) moiré pattern (5 $\times 10^{-7}$ Torr). (b) Relationship between the reciprocal lattice vectors. Here the filled circles represent the substrate lattice, the empty circles correspond to the adsorbate lattice, and the crosses correspond to the resulting moiré pattern. The observed vectors $\overrightarrow{\tilde{q}}_{\mathrm{M}}, \overrightarrow{\tilde{q}}_{\mathrm{S}}$, and $\overrightarrow{\tilde{q}}_{\mathrm{A}}$ are rotated by $30^{\circ}$ from the vectors $\vec{q}_{\mathrm{M}}, \vec{q}_{\mathrm{S}}$, and $\vec{q}_{\mathrm{A}}$ used in the analysis, and the vector lengths are related via $\tilde{q}_{x}=2 q_{x} / \sqrt{3}$, where $x=\mathrm{M}, \mathrm{S}$, A. The moiré vector $\overrightarrow{\tilde{q}}_{\mathrm{M}}$ is equal to the difference vector $\overrightarrow{\tilde{q}}_{\mathrm{M}}=\overrightarrow{\tilde{q}}_{\mathrm{A}}-$ $\overrightarrow{\tilde{q}}_{\mathrm{S}}$ and hence also $\vec{q}_{\mathrm{M}}=\vec{q}_{\mathrm{A}}-\vec{q}_{\mathrm{S}}$.

unreactive nature, ultrapure argon as commercially available is used for backfilling from the desired $\mathrm{CO}$ pressure up to the working pressure.

\section{Results}

Figure 1 shows two typical STM images of CO adsorbed on $\mathrm{Pt}(111)$ at room temperature. Image a was recorded at a $\mathrm{CO}$ pressure of $10^{-2}$ Torr, image $b$ at 720 Torr. In both images, two hexagonal structures coexist, one with a short and one with a longer periodicity. The short-periodicity hexagonal lattice is the image of either the $\mathrm{Pt}(111)$ substrate (panel a) or the CO overlayer (panel b), while the long-periodicity lattice (the moiré lattice) is characterized by the periodic height modulation induced by the superposition of the hexagonal $\mathrm{CO}$ adsorbate layer and the $\operatorname{Pt}(111)$ surface. ${ }^{16}$ Similar images, all of which exhibited moiré patterns, were obtained at the other investigated pressures.

We observed quite generally that we could not image the $\mathrm{CO}$ molecules at pressures below 0.1 Torr (at room temperature), but instead we sampled the underlying Pt(111) substrate, as in Figure 1a. The measurement of the moiré pattern with a hexagonal shape, however, proves the presence of a hexagonal $\mathrm{CO}$ overlayer. The invisibility of $\mathrm{CO}$ adsorbed on $\mathrm{Pt}$ and other metal surfaces in STM is known to occur under certain conditions (see, e.g., refs 18, 19, and 20).

Performing fast Fourier transforms (FFT) on the STM images allowed us to measure the periodicities and angles of the 


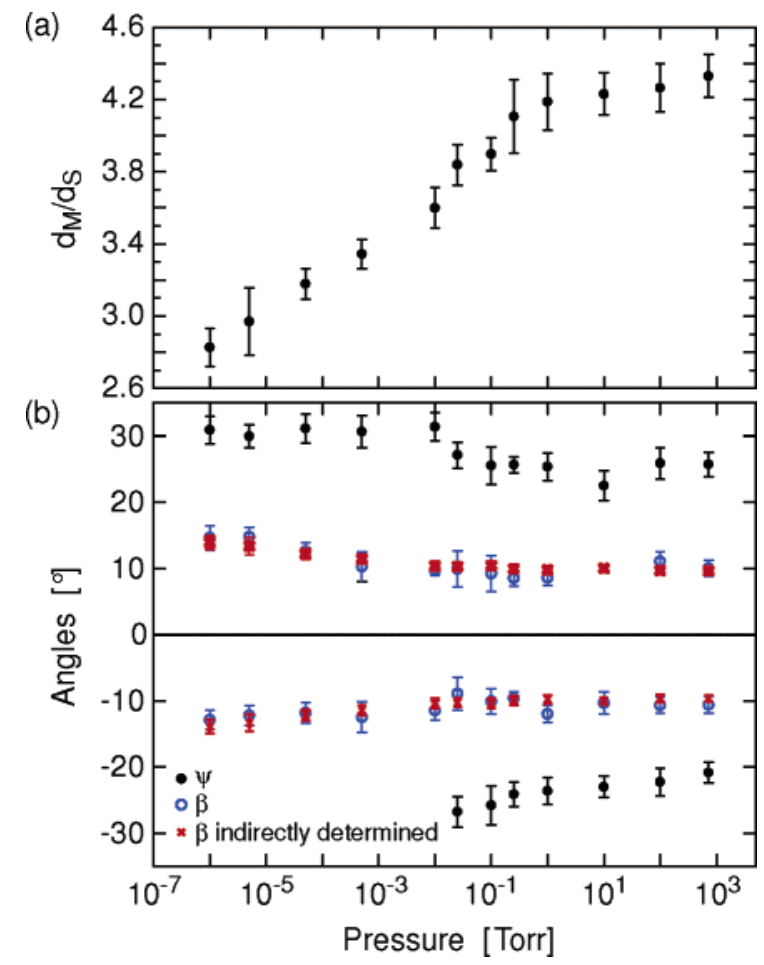

Figure 3. (a) Moiré lattice constant $d_{\mathrm{M}}$ for a $\mathrm{CO}$ layer on $\mathrm{Pt}(111)$ as a function of $\mathrm{CO}$ partial pressure. $d_{\mathrm{M}}$ is normalized with respect to the substrate lattice constant $d_{\mathrm{S}}$. (b) Rotational angles between the substrate and the moiré lattice $\Psi$ (filled symbols) and the substrate and adsorbate lattice $\beta$ (open symbols). $\beta$ can also be calculated from $d_{\mathrm{M}}, d_{\mathrm{S}}$, and $\Psi$ (crosses). ${ }^{21}$ In those cases, where two equivalent domains were observed (rotation of the moiré and adsorbate lattices by $\pm \Psi$ and $\pm \beta$ with respect to the substrate [110] direction, respectively), the angles for both domains are given.

observed structure. A typical FFT is shown in Figure 2a, and a sketch of the relevant parameters is shown in Figure $2 \mathrm{~b}$. These are the reciprocal lattice vectors $\overrightarrow{\tilde{q}}_{\mathrm{M}}$ of the moiré lattice, $\overrightarrow{\tilde{q}}_{\mathrm{A}}$ of the adsorbate lattice, and $\overrightarrow{\tilde{q}}_{\mathrm{S}}$ of the substrate lattice, which are related to each other by virtue of $\overrightarrow{\tilde{q}}_{\mathrm{M}}=\overrightarrow{\tilde{q}}_{\mathrm{A}}-\overrightarrow{\tilde{q}}_{\mathrm{S}}$. This set of reciprocal vectors $\overrightarrow{\tilde{q}}_{x}$ translates into the set $\vec{q}_{x}(x=\mathrm{M}, \mathrm{S}, \mathrm{A})$, where $\vec{q}_{x}$ are the reciprocal space vectors of the real space vectors $\vec{d}_{x}$ used in Figure 1 and in the analysis below, by a rotation of $30^{\circ}$ and by virtue of $\tilde{q}_{x}=2 q_{x} / \sqrt{3}$. Hence $\vec{q}_{\mathrm{M}}=\vec{q}_{\mathrm{A}}$ $-\vec{q}_{\mathrm{S}}$. Ball models showing the real space vectors are given in panels c and d of Figure 1. $\vec{d}_{\mathrm{M}}$ and $\vec{d}_{\mathrm{A}}$ form angles $\Psi$ and $\beta$, respectively, relative to the [1 $\overline{1} 0]$ direction of the substrate. From Figure $2 \mathrm{a}$, it is seen that the adsorbate lattice vector can be determined even if the adsorbates are not visible in the STM image, since the moiré spots appear as satellites around the substrate spots. ${ }^{21}$ The substrate spots can be identified unambiguously by comparison to the Fourier transforms of images taken on the clean $\operatorname{Pt}(111)$ crystal.

Figure 3 shows these parameters as a function of the $\mathrm{CO}$ partial pressure ranging from $10^{-6}$ to 760 Torr. In panel a, the moiré superlattice constant $d_{\mathrm{M}}$ is plotted in units of the $\operatorname{Pt}(111)$ substrate nearest neighbor distance $d_{\mathrm{S}}=2.77 \AA$. In panel b, the filled circles refer to the rotation angle $\Psi$ between the substrate and moire lattice and the open circles to the rotation angle $\beta$ between the substrate and adsorbate lattice. In addition, crosses indicate values for $\beta$ which were derived from the moiré parameters only as described in ref 21 . The procedure proved to be useful in case of barely visible satellites and gave additional confidence, since these values coincide with the $\beta$ values determined directly from the images within the measurement uncertainty. From Figure 3b, we can identify two regimes

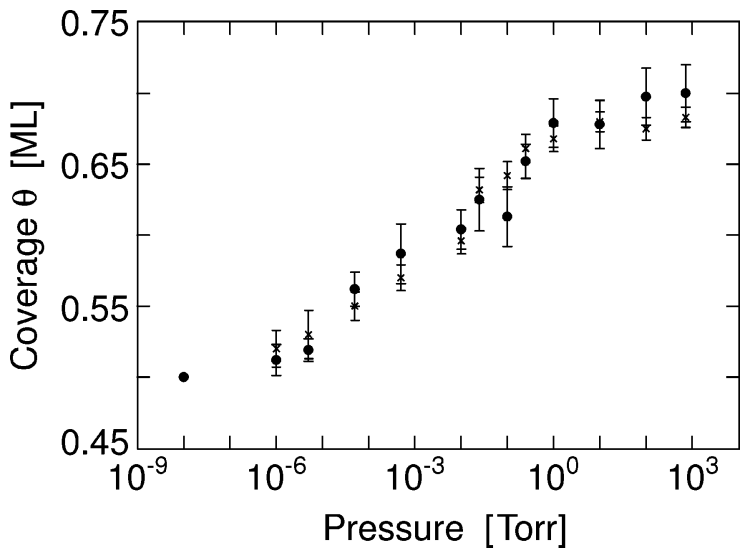

Figure 4. CO coverage $\theta$ on $\operatorname{Pt}(111)$ as a function of $\mathrm{CO}$ background pressure. The filled circles indicate values, which were directly determined from the STM images, while the indirectly determined values ${ }^{21}$ are indicated by crosses.

with different types of incommensurate moiré superstructures. In the pressure range from $10^{-6}$ to $10^{-2}$ Torr, we observe a moiré pattern, which is rotated by a fixed angle of $\Psi=30^{\circ}$ with respect to the $\operatorname{Pt}(111)$ substrate, whereas the adsorbate angle $\beta$ decreases from about 14 to $10^{\circ}$. At the same time, the lattice constant of the moiré pattern changes approximately linearly with the logarithm of the $\mathrm{CO}$ pressure. At $\mathrm{CO}$ pressures above $10^{-2}$ Torr, $\Psi$ decreases, while $\beta$ remains approximately constant at about $10^{\circ}$. Close to atmospheric pressure, the moiré lattice constant $d_{\mathrm{M}}$ approaches a fixed saturation value.

In panels $\mathrm{c}$ and $\mathrm{d}$ of Figure 1, a schematic model of the $\mathrm{CO}$ structures observed at $10^{-2}$ and 720 Torr is given. The ball model in panel c was created with the $\Psi, d_{\mathrm{M}}, d_{\mathrm{A}}$, and $\beta$ values from the measurement. The structure at 720 Torr is compatible with a $(\sqrt{19} \times \sqrt{19}) \mathrm{R} 23.4^{\circ}-13 \mathrm{CO}$ commensurate structure, and the corresponding values were used in panel $\mathrm{d}$.

Knowledge of the substrate and adsorbate lattice constants allows one also to determine the $\mathrm{CO}$ coverage, which is given by $\theta=\left(d_{\mathrm{S}} / d_{\mathrm{A}}\right)^{2}=\left(q_{\mathrm{A}} / q_{\mathrm{S}}\right)^{2}$. Figure 4 shows how the coverage increases continuously with $\mathrm{CO}$ pressure in the range of $10^{-8}-$ $10^{3}$ Torr. Toward atmospheric pressures, the $\mathrm{CO}$ coverage saturates due to the increasingly repulsive $\mathrm{CO}-\mathrm{CO}$ interaction. We confirmed that the variation of the $\mathrm{CO}$ coverage was reversible by first exposing the sample to a $\mathrm{CO}$ pressure of 760 Torr and then adjusting the pressure to a lower value. The structural parameters were the same as for a sample that was exposed to the lower pressure only. The results for the $\mathrm{CO}$ coverage are in good agreement with the previously determined CO coverages at $10^{-8}$ Torr $(\theta=0.5)^{3}$ and 760 Torr $(\theta=0.68) .{ }^{10}$ The CO coverage given for $10^{-8}$ Torr corresponds to the $\mathrm{c}(4 \times$ 2) structure observed in low-energy electron diffraction (LEED). ${ }^{3,22}$

\section{Discussion}

From Figure 4, the coverage of $\mathrm{CO}$ on $\mathrm{Pt}(111)$ at room temperature is seen to vary continuously (and reversibly, as outlined above) over the pressure range from $10^{-6}$ to 760 Torr. The observed CO structures were hexagonally ordered. The finding of a continuous variation of the $\mathrm{CO}$ coverage with pressure and the observation of hexagonal, ordered overlayer structures for all pressures above $10^{-6}$ Torr is in contrast to the $\mathrm{CO} / \mathrm{Pt}(111)$ lattice gas structures found in the same coverage range at lower pressures and temperatures., 3,12 The formation of lattice gas structures would lead to a phase diagram with alternating regimes of ordered and disordered $\mathrm{CO}$ overlayers. ${ }^{4}$ 


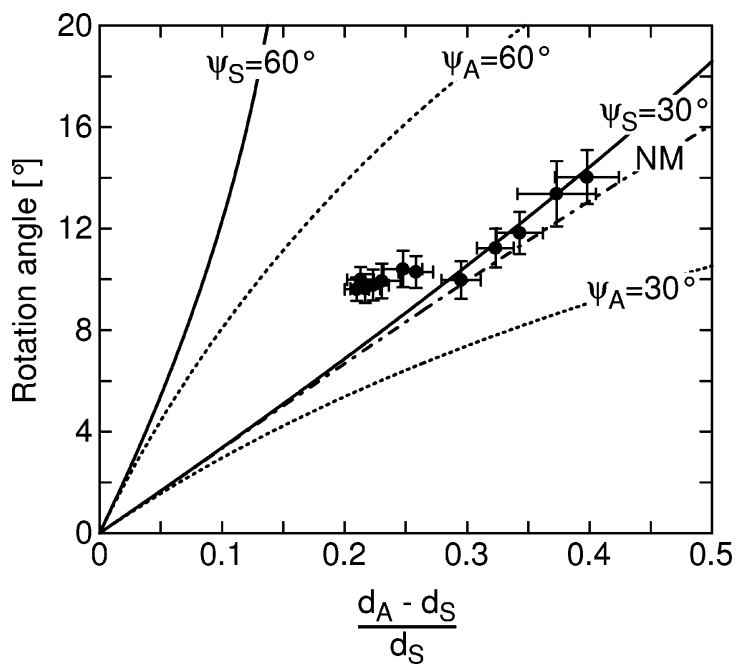

Figure 5. Rotation angle between $\mathrm{CO}$ adsorbate layer and $\mathrm{Pt}(111)$ substrate as a function of the lattice misfit between $d_{\mathrm{A}}$ and $d_{\mathrm{S}},\left(d_{\mathrm{A}}-\right.$ $\left.d_{\mathrm{s}}\right) / d_{\mathrm{s}}$. The solid and short-dashed lines indicate the theoretical highsymmetry solutions of ref 35 and the dashed-dotted line marked NM the harmonic approximation of ref 29 for a Lennard-Jones system. $\Psi_{\mathrm{S}}$ is the rotation angle of the moiré superlattice with respect to the substrate. $\Psi_{\mathrm{A}}$ is the rotation angle of the moiré superlattice with respect to the adsorbate.

Nevertheless, a true pressure gap is not observed for the $\mathrm{CO} /$ $\mathrm{Pt}(111)$ system since cooling of the sample to approximately $170 \mathrm{~K}$ leads to an increase in $\mathrm{CO}$ coverage and the formation of a hexagonal $\mathrm{CO}$ overlayer similar to that at 760 Torr. ${ }^{10}$

The formation of moiré structures has been observed previously for a large variety of adsorbate systems. For example, moiré patterns for $\mathrm{CO}$ overlayers have been reported for the $\mathrm{Ni}(111),{ }^{23} \mathrm{Ag}(111),{ }^{24}$ and graphite ${ }^{25}$ substrates and, at a pressure of $\sim 760$ Torr, also for the $\mathrm{CO} / \mathrm{Pt}(111)$ system., ${ }^{710}$ Generally, the occurrence of moiré patterns can always be expected when the adsorbate lateral repulsive potential $V_{1}$ balances or exceeds the corrugation $\Delta U$ of the adsorbate-substrate interaction potential. In the $V_{1} \gg \Delta U$ and infinite lattice limit, the resulting structure can be viewed as a mere superposition of two perfect, in this case hexagonal, lattices of the substrate and adsorbate, respectively. Because of the small difference in the adsorbate and substrate lattice constants, a long-periodicity (given by the moiré wave vector) height modulation is then observed. ${ }^{16}$

In the case $V_{1} \approx \Delta U$, the situation is more complicated and has received considerable theoretical interest. ${ }^{26-36}$ The various theories predict an alignment of the moiré vector in preferred directions if the molecules are able to relax their positions with respect to the perfect hexagonal overlayer lattice ${ }^{26-32}$ and/or if the system has a finite size..$^{3-36}$ References $33-36$ put forward a "high-symmetry hypothesis" according to which the adsorbate layer rotates by an angle $\beta$ such that the resulting incommensurate moiré superlattice is either oriented along a highsymmetry direction of the substrate or along a high-symmetry direction of the adsorbate layer. Numerical calculations showed that these situations correspond to an energy minimum, since the number of adsorbates in or close to low-energy sites is maximized. ${ }^{34,35,36}$ The maximization is solely due to a finitesize effect, with relaxation only resulting in a second-order correction.

In Figure 5 the rotation angle $\beta$ of the $\mathrm{CO}$ lattice with respect to the Pt substrate as a function of the lattice misfit $\left(d_{\mathrm{A}}-d_{\mathrm{S}}\right)$ / $d_{\mathrm{S}}$ is compared to the high-symmetry ( 30 and $60^{\circ}$ ) solutions relevant for the (111) surface. For comparison, we also show, marked NM, the theoretical harmonic approximation ${ }^{37}$ solution

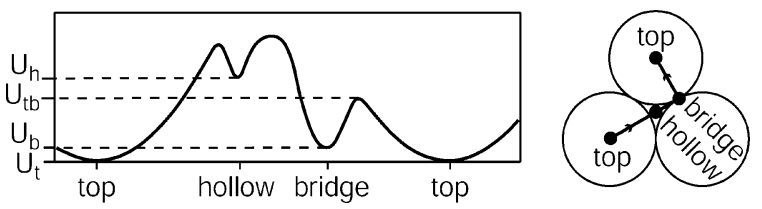

Figure 6. Qualitative potential energy curve for an isolated $\mathrm{CO}$ molecule on $\operatorname{Pt}(111)$ along the lines connecting the top, bridge and hollow sites.

derived for a Lennard-Jones system in refs 26 and 29. (The longitudinal and transverse sound velocities $c_{\mathrm{L}}$ and $c_{\mathrm{T}}$ are the only system parameters which enter into the solution. For a twodimensional hexagonal lattice they are related to each other by $c_{\mathrm{L}}=\sqrt{3} c_{\mathrm{T}}$.) For misfit values between 0.3 and 0.4 , i.e., coverages below 0.6 monolayers, the experimental data points are found to be in good agreement with the $\Psi_{\mathrm{S}}=30^{\circ}$ solution found from the high-symmetry hypothesis, i.e., the moiré vector aligns with the $30^{\circ}$ high-symmetry direction of the substrate (which corresponds to the [121] direction). For misfit values lower than 0.3 , however, a deviation from the theoretical lines is observed. As the compression of the $\mathrm{CO}$ layer continues, i.e., for lower values of $\left(d_{\mathrm{A}}-d_{\mathrm{S}}\right) / d_{\mathrm{S}}, \beta$ remains constant at approximately $10^{\circ}$ within the error bars, whereas $\Psi$ decreases from $30^{\circ}$ to about $\pm 24^{\circ}$ (cf. Figure $3 b$ ).

The alignment of the moiré vector with the $30^{\circ}$ substrate direction indicates that the particular rotation of the adsorbate layer is due to the finite size of the substrate. ${ }^{35,36}$ The role of the substrate boundaries is played by the substrate steps, which are oriented along the [1 $1 \overline{0}]$ and equivalent directions. It would be interesting to confirm this result in experiments using samples with varying step density.

As established above, the moiré vector does not align with any of the substrate high-symmetry directions at pressures above $10^{-2}$ Torr. The energy gain associated with this transition (see discussion below) thus does not give a sufficiently high impetus to achieve the rotation. We can analyze the situation in further detail by taking the form of the substrate interaction potential $U$ and the strength of the lateral interaction $V_{1}$ into account. The exact form of $V_{1}$ has not yet been determined (see refs 3841 ), but an approximation is given by the semiempirical CO$\mathrm{CO}$ pairwise interaction potential in ref 42 . A qualitative sketch of $U$ is given in Figure 6, and Table 1 reproduces some potential parameters, derived from the semiempirical single-molecule potential curve ${ }^{43}$ of ref 42 . The top sites are energetically most favorable, followed by the bridge sites. ${ }^{3-5}$ Here we assume that the maximum of the potential energy barrier $U_{\mathrm{tb}}$ between top and bridge sites lies below the local potential energy minimum $U_{\mathrm{h}}$ at hollow sites, in agreement with the calculations in ref 38 and the experimental findings of ref 11 . As a consequence, the hollow sites are not occupied over the entire investigated pressure range, in agreement with the results of ref 11 . The observation of a hexagonal superstructure is not contradicted, since we expect the positions of the adsorbed $\mathrm{CO}$ molecules to be relaxed with respect to a perfect hexagonal overlayer. In addition, the area of the near-hollow sites amounts to a relatively small fraction of the total surface area only, so that these sites do not necessarily have to be occupied in a close-to-hexagonal overlayer.

Relevant for a comparison of the lateral interaction energy and the corrugation of the substrate potential are the barriers between the different sites. In the analysis, we have to take into account that the measurements are carried out at room temperature. A crude estimation for the effective barrier height is derived by assuming parabolic and isotropic oscillators. The resulting values for the effective barriers between the bridge 
TABLE 1: Parameters of the Employed Substrate Interaction Potential and the Lateral Interaction (All Energies Are Given in $\mathrm{meV})^{a}$

\begin{tabular}{rccccccccc}
\hline$T(\mathrm{~K})$ & $U_{\mathrm{t}}$ & $U_{\mathrm{b}}$ & $U_{\mathrm{tb}}$ & $\left\langle U_{\mathrm{t}}\right\rangle$ & $\left\langle U_{\mathrm{b}}\right\rangle$ & $\begin{array}{c}\Delta U_{\mathrm{tb}-\mathrm{t}} \equiv U_{\mathrm{tb}} \\
-\left\langle U_{\mathrm{t}}\right\rangle-U_{\mathrm{t}}\end{array}$ & $\begin{array}{c}\Delta U_{\mathrm{tb}-\mathrm{b}} \equiv U_{\mathrm{tb}}-\left\langle U_{\mathrm{b}}\right\rangle \\
-U_{\mathrm{b}}\end{array}$ & $V_{\mathrm{l}}(\theta=0.5)$ & $V_{\mathrm{l}}(\theta=0.6)$ \\
\hline 0 & 0 & 60 & 300 & 6 & 37 & 294 & 203 & 200 \\
300 & 0 & 60 & 300 & 72 & 60 & 248 & 180 & 200 \\
\end{tabular}

${ }^{a} \Delta U_{\mathrm{tb}-\mathrm{t}}$ and $\Delta U_{\mathrm{tb}-\mathrm{b}}$ represent effective barrier heights relative to the mean energy of the molecule inside the potential wells, which were assumed to be parabolic. These effective barrier heights are derived assuming isotropic oscillators with energies $\left\langle U_{\mathrm{t}, \mathrm{b}}\right\rangle=\hbar \omega_{\mathrm{t}, \mathrm{b}}+\hbar \omega_{\mathrm{t}, \mathrm{b}} /\left(\exp \left(\hbar \omega_{\mathrm{t}, \mathrm{b}} / k T\right)-1\right)$, where $\hbar \omega_{\mathrm{t}}=48 \mathrm{~cm}^{-1}$ and $\hbar \omega_{\mathrm{b}}=300 \mathrm{~cm}^{-1}$ are the vibrational motion frequencies in the top and bridge positions, respectively. ${ }^{42}$ The values defining the potentials $\left(U_{\mathrm{t}}, U_{\mathrm{b}}, U_{\mathrm{tb}}\right.$, and $\left.V_{\mathrm{l}}\right)$ have been taken to be temperature independent.

and on-top sites, $\Delta U_{\mathrm{tb}-\mathrm{t}} \equiv U_{\mathrm{tb}}-\left\langle U_{\mathrm{t}}\right\rangle-U_{\mathrm{t}}$, as seen from the on-top potential-energy minimum and $\Delta U_{\mathrm{tb}-\mathrm{b}} \equiv U_{\mathrm{tb}}-\left\langle U_{\mathrm{b}}\right\rangle-$ $U_{\mathrm{b}}$ as seen from the bridge minimum, are given in Table 1. $\Delta U_{\mathrm{tb}-\mathrm{t}}$ and $\Delta U_{\mathrm{tb}-\mathrm{b}}$ represent the room-temperature effective barrier heights relative to the mean energy of the molecule inside the potential wells (see the Table caption), which were assumed to be parabolic, while $U_{\mathrm{t}}, U_{\mathrm{b}}$, and $U_{\mathrm{tb}}$ are the parameters of the potential energy surface for the on-top and bridge sites and the barrier between these (cf. Figure 6). ${ }^{42}$ For the on-top sites, the lateral repulsion energy $V_{1}$ is smaller than the barrier height $\Delta U_{\mathrm{tb}-\mathrm{t}}$ at the onset of the high-symmetry moiré phase with $\theta$ $\approx 0.5$. At $\theta \approx 0.6$, corresponding to the rotational transition at $10^{-2}$ Torr, $V_{1}$ is essentially equal to $\Delta U_{\mathrm{tb}-\mathrm{t}}$. In contrast, the barrier as seen from the bridge sites, $\Delta U_{\mathrm{tb}-\mathrm{b}}$, is found to be smaller than the lateral repulsion $V_{1}$ over the entire coverage range of $0.5-0.6$. This indicates that the occupation of top sites is decisive for the formation of the high-symmetry moiré phase at lower $\mathrm{CO}$ pressures.

At pressures above $10^{-2}$ Torr, the orientation of the moiré superstructure along the high-symmetry direction $\Psi=30^{\circ}$ is not maintained. Hence, the rotation concomitant with such an alignment is not favorable anymore, and instead, the $\mathrm{CO}$ adsorbate layer keeps a constant orientation with respect to the substrate. In this pressure regime, the lateral repulsion exceeds the barrier height between the on-top and bridge position, irrespective of whether these are measured with reference to the bridge $\left(\Delta U_{\mathrm{tb}-\mathrm{b}}\right)$ or on-top site $\left(\Delta U_{\mathrm{tb}-\mathrm{t}}\right)$. Hence the tendency of the CO molecules to occupy (near) top sites is reduced. We therefore propose that the increasing dominance of the lateral repulsion over the barrier height $\Delta U_{\mathrm{tb}-\mathrm{t}}$ causes the deviation from the high-symmetry direction just above $10^{-2}$ Torr.

However, close to 760 Torr, the occupation of top sites again becomes decisive, namely, for the formation of a commensurate $(\sqrt{19} \times \sqrt{19}) \mathrm{R} 23.4^{\circ}-13 \mathrm{CO}$ structure. In this commensurate structure, a very high number of $\mathrm{CO}$ molecules can sit exactly on top sites. We note that the electrochemical study of Villegas and Weaver ${ }^{11}$ indicates that going from a negative to a slightly positive sample bias leads to a switching from a $(2 \times 2)-3 \mathrm{CO}$ structure to the $(\sqrt{19} \times \sqrt{19}) \mathrm{R} 23.4^{\circ}-13 \mathrm{CO}$ system. The rotation concomitant with such a transformation indicates an enhanced stability of the $(\sqrt{19} \times \sqrt{19})$ structure. Together with the present results, this finding suggests that the occupancy of a high number of on-top sites to some extent can outweigh the lateral repulsion even at high coverages.

The energy gain due to the formation of the commensurate $(\sqrt{19} \times \sqrt{19}) \mathrm{R} 23.4^{\circ}-13 \mathrm{CO}$ structure is judged to be higher than the energy gain that would result from a reorientation of the incommensurate moiré superstructure along $\Psi=30^{\circ}$ just above $10^{-2}$ Torr. We suggest that above $10^{-2}$ Torr the lateral forces are sufficient to exceed the corrugation of the substrate potential. Hence a simple reorientation along a high-symmetry direction is prevented but not the formation of a particularly stable commensurate structure.

\section{Summary}

In conclusion, we have determined the room-temperature surface structure of $\mathrm{CO}$ on $\mathrm{Pt}(111)$ over the entire pressure range of $10^{-6}-760$ Torr. For all pressures, the formation of a hexagonal or quasihexagonal $\mathrm{CO}$ overlayer is observed, showing that for $\mathrm{CO} / \mathrm{Pt}(111)$ a true pressure gap cannot be established. Nevertheless, extreme care has to be taken when relating UHV results to systems operating under realistic conditions such as atmospheric pressure. One has to consider (a) the existence of different commensurate vacuum structures on cooled samples (see, e.g., refs 3 and 4) and (b) the subtle differences between the reported hexagonal structures in terms of the adsorbate layer rotation angle.

In further detail, the $\mathrm{CO}$ adsorbate layer is continuously compressed with increasing pressure, resulting in a continuous coverage variation from 0.5 to 0.7 . The orientation of the moiré superlattice, whose formation is due to the superposition of the hexagonal adsorbate layer with the hexagonal substrate, is pressure dependent. First, for coverages between $\sim 0.5$ and 0.6 , the moire pattern is rotated by $30^{\circ}$ with respect to the [1 10$]$ direction of the $\operatorname{Pt}(111)$ substrate. The orientation can be explained in terms of a maximum occupation of high-binding energy sites for a substrate of finite size, ${ }^{35,36}$ where the size is given by the surface terraces. Second, at a coverage of 0.6 , the system undergoes a rotational phase transition. Above this coverage, the rotation angle of the adsorbate layer with respect to the substrate does not change anymore. We explain this behavior in terms of an increasing importance of the repulsive lateral molecular interaction, which starts to dominate over the corrugation of the substrate interaction potential. A quantitative analysis based on previous results ${ }^{42}$ supports our explanation.

\section{References and Notes}

(1) Handbook of Heterogeneous Catalysis; Ertl, G., Knözinger, H., Weitkamp, J., Eds.; Wiley-VCH: Weinheim, 1997; Vols. 1-5.

(2) See, e.g., ref 1, Vol. 4, p 2094.

(3) Ertl, G.; Neumann, M.; Streit, K. M. Surf. Sci. 1977, 64, 393.

(4) Persson, B. N. J.; Tüshaus, M.; Bradshaw, A. M. J. Chem. Phys. 1990, 92, 5034 .

(5) McEwen, J.-S.; Payne, S.; Kreuzer, H. J.; Kinne, M.; Denecke, R.; Steinrück, H.-P. Surf. Sci. 2003, 545, 47.

(6) Su, X.; Cremer, P. S.; Ron Shen, Y.; Somorjai, G. A. Phys. Rev. Lett. 1996, 77, 3858.

(7) Jensen, J. A.; Rider, K. B.; Salmeron, M.; Somorjai, G. A. Phys. Rev. Lett. 1998, 80, 1228.

(8) Ertl, G. Angew. Chem., Int. Ed. Engl. 1990, 29, 1219.

(9) Rupprechter, G.; Dellwig, T.; Unterhalt, H.; Freund, H.-J. J. Phys. Chem. B 2001, 105, 3797.

(10) Kruse Vestergaard, E.; Thostrup, P.; An, T.; Lægsgaard, E.; Stensgaard, I.; Hammer, B.; Besenbacher, F. Phys. Rev. Lett. 2002, 88, 259601 .

(11) Villegas, I.; Weaver, M. J. J. Chem. Phys. 1994, 101, 1648.

(12) Biberian, J. P.; van Hove, M. A. Surf. Sci. 1984, 138, 361.

(13) Here we define the coverage $\theta$ as the number of $\mathrm{CO}$ molecules per substrate surface atom.

(14) Besenbacher, F. Rep. Prog. Phys. 1996, 59, 1737

(15) Lægsgaard, E.; Österlund, L.; Thostrup, P.; Rasmussen, P. B.; Stensgaard, I.; Besenbacher, F. Rev. Sci. Instrum. 2001, 72, 3537. 
(16) Note that the observation of a moire pattern in STM in general is due to a combination of electronic and geometric effects. In fact, it has been argued that the imaging of moiré patterns in STM is not due to the modulation of the topography, but to (three-dimensional) tunneling from the buried interface. ${ }^{17}$ In this model, the enhanced visibility of the moire pattern is explained in terms of the larger decay length of the long-periodicity wave function, which is associated with the moire lattice and which is produced by multiple scattering. The exact imaging mechanism is not important in the present context though, since the presence of a moiré pattern unambiguously proves the presence of a (nearly) hexagonal $\mathrm{CO}$ adsorbate layer.

(17) Kobayashi, K. Phys. Rev. B 1996, 53, 11091

(18) Ramos, M. M. D.; Stoneham, A. M.; Sutton, A. P.; Pethica, J. B. J. Phys.: Condens. Matter 1990, 2, 5913.

(19) Thostrup, P.; Kruse Vestergaard, E.; An, T.; Lægsgaard, E.; Besenbacher, F. J. Chem. Phys. 2003, 118, 3724.

(20) Sprunger, P. T.; Besenbacher, F.; Stensgaard, I. Chem. Phys. Lett. 1995, 243, 439

(21) In some cases, the satellites were not visible in the STM. In these cases, and as an additional confirmation to the others, the CO lattice parameters can be inferred from the measured moiré superlattice parameters. From Figure 2b, one can derive the following formula:

$$
\begin{gathered}
q_{\mathrm{A}}=\left(q_{\mathrm{S}}{ }^{2}+q_{\mathrm{M}}{ }^{2}-2 q_{\mathrm{S}} q_{\mathrm{M}} \cos (\pi-\alpha)\right)^{1 / 2} \\
\sin \beta=\frac{q_{\mathrm{M}}}{q_{\mathrm{A}}} \sin (\pi-\alpha) \\
\pi-\alpha=\frac{\pi}{3}-\Psi
\end{gathered}
$$

$\Psi$ is taken to be the smallest positive angle between the substrate and moiré lattices. This set of equations allows a determination of all relevant angles and lattice vectors.

(22) The LEED images observed by Ertl et al. ${ }^{3}$ exhibit clear spots at temperatures below and more diffuse spots at room temperature, which points to some degree of overlayer disorder at room temperature. The clear LEED pattern can be recovered by sample cooling, and hence the coverage is the same in both cases $(\theta=0.5)$.

(23) Quiros, C.; Robach, O.; Isern, H.; Ordejon, P.; Ferrer, S. Surf. Sci. 2003, 522, 161 .

(24) Leatherman, G. S.; Diehl, R. D. Langmuir 1997, 13, 7063.
(25) Steele, W. Chem. Rev. 1993, 93, 2355.

(26) Novaco, A. D.; McTague, J. P. Phys. Rev. Lett. 1977, 38, 1286.

(27) Villain, J. Phys. Rev. Lett. 1978, 41, 36

(28) Novaco, A. D. Phys. Rev. B 1979, 19, 6493.

(29) McTague, J. P.; Novaco, A. D. Phys. Rev. B 1979, 19, 5299.

(30) Shiba, H. J. Phys. Soc. Jpn. 1979, 46, 1852.

(31) Shiba, H. J. Phys. Soc. Jpn. 1980, 48, 211.

(32) Villain, J.; Gordon, M. B. Surf. Sci. 1983, 125, 1.

(33) Grey, F.; Bohr, J. In Phase Transitions in Surface Films 2; Taub, H., Torzo, G., Lauter, H., Fain, S. C., Eds.; Plenum Press: New York, 1991; NATO ASI Series, Series B, Physics, Vol. 267, p 83.

(34) Grey, F.; Bohr, J. Appl. Surf. Sci. 1993, 65-66, 35

(35) Grey, F.; Bohr, J. Europhys. Lett. 1992, 18, 717.

(36) Bohr, J.; Grey, F. Condens. Matter News 1992, 1, 12.

(37) Anharmonic extensions of the theory of Novaco and McTague have also been formulated, see refs 27,28 , and 32 .

(38) van Beurden, P.; Verhoeven, H. G. J.; Kramer, G. J.; Thijsse, B. J. Phys. Rev. B 2002, 66, 235409.

(39) Jennison, D. R.; Schultz, P. A.; Sears, M. P. Phys. Rev. Lett. 1996, $77,4828$.

(40) Brako, R.; Šokčević, D. Surf. Sci. 2000, 469, 185.

(41) Petrova, N.; Yakovkin, I. Surf. Sci. 2002, 519, 90.

(42) Schweizer, E.; Persson, B. N. J.; Tüshaus, M.; Hoge, D.; Bradshaw, A. M. Surf. Sci. 1989, 213, 49.

(43) Ref 44 indicates that for $\mathrm{CO} / \mathrm{Pt}(111)$ density functional calculations (DFT) deliver qualitatively wrong results for the potential-energy surface. While this statement subsequently has been softened ${ }^{45-48}$ or disputed, ${ }^{49}$ DFT results are not reliable for our purposes, and we have to use results from less advanced methods.

(44) Feibelman, P. J.; Hammer, B.; Nørskov, J. K.; Wagner, F.; Scheffler, M.; Stumpf, R.; Watwe, R.; Dumesic, J. J. Phys. Chem. B 2001, 105, 4018.

(45) Grinberg, I.; Yourdshahyan, Y.; Rappe, A. M. J. Chem. Phys. 2002 117,2264

(46) Geschke, D.; Baştuğ, T.; Jacob, T.; Fritzsche, S.; Sepp, W.-D.; Fricke, B.; Varga, S.; Anton, J. Phys. Rev. B 2001, 64, 235411.

(47) Gil, A.; Clotet, A.; Ricart, J. P.; Kresse, G.; García-Hernández, M.; Rösch, N.; Sautet, P. Surf. Sci. 2003, 530, 71.

(48) Kresse, G.; Gil, A.; Sautet, P. Phys. Rev. B 2003, 68, 073401.

(49) Olsen, R. A.; Philipsen, P. H. T.; Baerends, E. J. J. Chem. Phys. 2003, 119, 4522 . 